\title{
Consumo de plantas pioneras por murciélagos frugívoros en un fragmento de bosque seco tropical (Colombia)
}

\section{Pioneer plants consumption by frugivorous bats in a fragment of tropical dry forest (Colombia)}

\author{
Leidy Viviana García-Herrera ${ }^{a^{*}}$ \\ Leidy Azucena Ramírez-Fráncel ${ }^{\text {a }}$ \\ Gladys Reinoso-Flórez ${ }^{\mathrm{b}}$
}

Fecha de Recepción: 22.07.18

Fecha de Aceptación: 10.06.19

DOI: https://doi.org/10.19053/01217488.v10.n2.2019.8240

\begin{abstract}
Resumen
La dispersión de semillas juega un papel importante en la regeneración y el mantenimiento de los bosques; ya que, mediante este mecanismo, las plantas encuentran la posibilidad de colonizar nuevos sitios y mantener la estructura poblacional. Los murciélagos han sido reconocidos como dispersores efectivos en varios ecosistemas tropicales, debido a su capacidad de defecar en vuelo, sobrevolar áreas abiertas y ejercer un efecto de germinación sobre las semillas liberadas. Con base en lo anterior, se evaluó la preferencia en la dieta de un ensamble de murciélagos frugívoros del bosque seco tropical del departamento del Tolima. El análisis de 63 registros dietarios de 14 especies de murciélagos permitió identificar 11 especies de plantas, tres géneros y una morfoespecie, donde Bidens pilosa, Piper crassinervium y Physalis peruviana L. presentaron el mayor consumo. Artibeus lituratus registro la mayor amplitud de dieta seguido por Artibeus planirostris. Se presentó una baja sobreposiciones de dieta, lo que podría reflejar una segregación de nicho, como estrategia para evitar la competencia entre especies simpátricas. Se resalta el consumo de frutos en estado sucesional arbusto, lo cual reafirma el papel clave de los murciélagos en la dispersión de especies pioneras.
\end{abstract}

Palabras clave: Chiroptera, Bosque seco tropical, frugivoría, semillas.

a $\mathrm{PhD}(\mathrm{c})$ en Ciencias Biológicas, MSc. en Ciencia Biológicas, Grupo de Investigación en Zoología, Facultad de Ciencias, Universidad del Tolima.

b MSc. en Ciencia Biológicas, Grupo de Investigación en Zoología, Facultad de Ciencias, Universidad del Tolima.

* Autor de Correspondencia: lvgarcia@ut.edu.co 


\begin{abstract}
Seed dispersal is an important process for the regeneration and maintenance of forests; This mechanism allows plants to colonize new sites and maintain the structure of the population. Bats have been recognized as effective dispersers of seeds in several tropical forest ecosystems, due to the ability to defecate in flight, fly over open areas and exert a germination effect on released seeds. In this study was to evaluate diet preference of fruit bats in a tropical dry forest in the department of Tolima. The analysis of 63 dietary records of 14 bat species allowed to identify 11 plant species, three genera and one morphospecies. Bidens pilosa, Piper crassinervium and Physalis peruviana L. showed the highest consumption. Artibeus lituratus had the widest niche breath, followed by Artibeus planirostris. Low overlays were present among these bat species, which could reflect a niche segregation as a strategy to avoid competition between sympatric species. The consumption of fruits in a successional shrubs state is highlighted, which reaffirms the key role of bats in the dispersion of pioneer species.
\end{abstract}

Keywords: Chiroptera, Frugivory, tropical dry forest, seed.

\section{INTRODUCCIÓN}

Los frugívoros son ecológicamenteimportantes como dispersores de semillas [1], servicio ecosistémico fundamental para la dinámica y regeneración de los bosques, especialmente en las zonas tropicales, en donde un número importante de plantas dependen de los animales para su reproducción [2]. Se estima que cerca del $80 \%$ de las especies de plantas en el Neotrópico dependen de los vertebrados frugívoros para la dispersión de sus diásporas, contrarrestando la endogamia $\mathrm{y}$ favoreciendo el intercambio genético entre poblaciones [3].

Entre los vertebrados, los murciélagos han sido reconocidos como importantes dispersores de especies de sucesión temprana y pionera [4], proceso que se ve favorecido por su capacidad de sobrevolar áreas abiertas, defecar durante el vuelo, presentar diferentes estrategias de forrajeo, amplia gama dietaría $[4,5]$ y efecto de germinación sobre los propágulos liberados, ya que el paso de las semillas a través del intestino a menudo reduce la mortalidad y favorece su crecimiento [6], factores que posicionan a los murciélagos como importantes dispersores de semillas en ecosistemas tropicales [7].

El Bosque Seco Tropical (bs-T) alberga un alto endemismo y diversidad [8], características que, junto con la intensa transformación por actividades antropogénicas, lo constituye en una prioridad para la conservación [9]. En Colombia la distribución actual del bs-T está directamente relacionada con los procesos de deforestación y colonización que se han desarrollado en el país, lo que ha generado una alta modificación, encontrándose en fragmentos de bosque rodeado por matrices de cultivos, pastos o matorrales [10], situación que atenta contra la biodiversidad asociada, los procesos ecológicos que aseguran su funcionabilidad y los servicios ecosistémicos que proveen [11].

La relación mutualista, murciélago-planta y los servicios ecológicos que esta fauna provee han sido poco estudiada en los bosques secos, particularmente en Colombia, donde los estudios se han centrado en los departamentos de Córdoba, Caldas y Meta [12, 13, 14], existiendo vacíos de información en otras regiones como el alto valle del río Magdalena, áreas de interés de conservación. Por lo tanto, el objetivo de esta investigación fue evaluar la preferencia de dieta de un ensamble de murciélagos frugívoros del bs-T del departamento del Tolima, identificando la amplitud de dieta, importancia de cada especie como dispersor y nivel de solapamiento de nicho trófico.

\section{METODOLOGÍA}

El estudio se realizó en un fragmento de 18 ha de bs-T nativo en la hacienda el Edén ubicada en el extremo norte del municipio de San Sebastián de Mariquita, en el departamento del Tolima, Colombia (5¹7'10.86”N 7451'30.08'O). El área se caracteriza por presentar un clima cálido y húmedo, con una altura entre los 300 y 1,200 m.s.n.m. La distribución de lluvias es de carácter bimodal, con un promedio anual de 2,409 $\mathrm{mm}$, 
temperatura media de $26.5^{\circ} \mathrm{C}$ y humedad relativa del $82 \%$ [15]. La vegetación característica del área de estudio comprende bosque natural con una vegetación leñosa, arbórea y arbustiva de gran variedad de especies florísticas que tiene como finalidad la protección de márgenes y nacimientos de microcuencas, así como el control de los procesos erosivos en zonas de altas [15] (Figura 1).

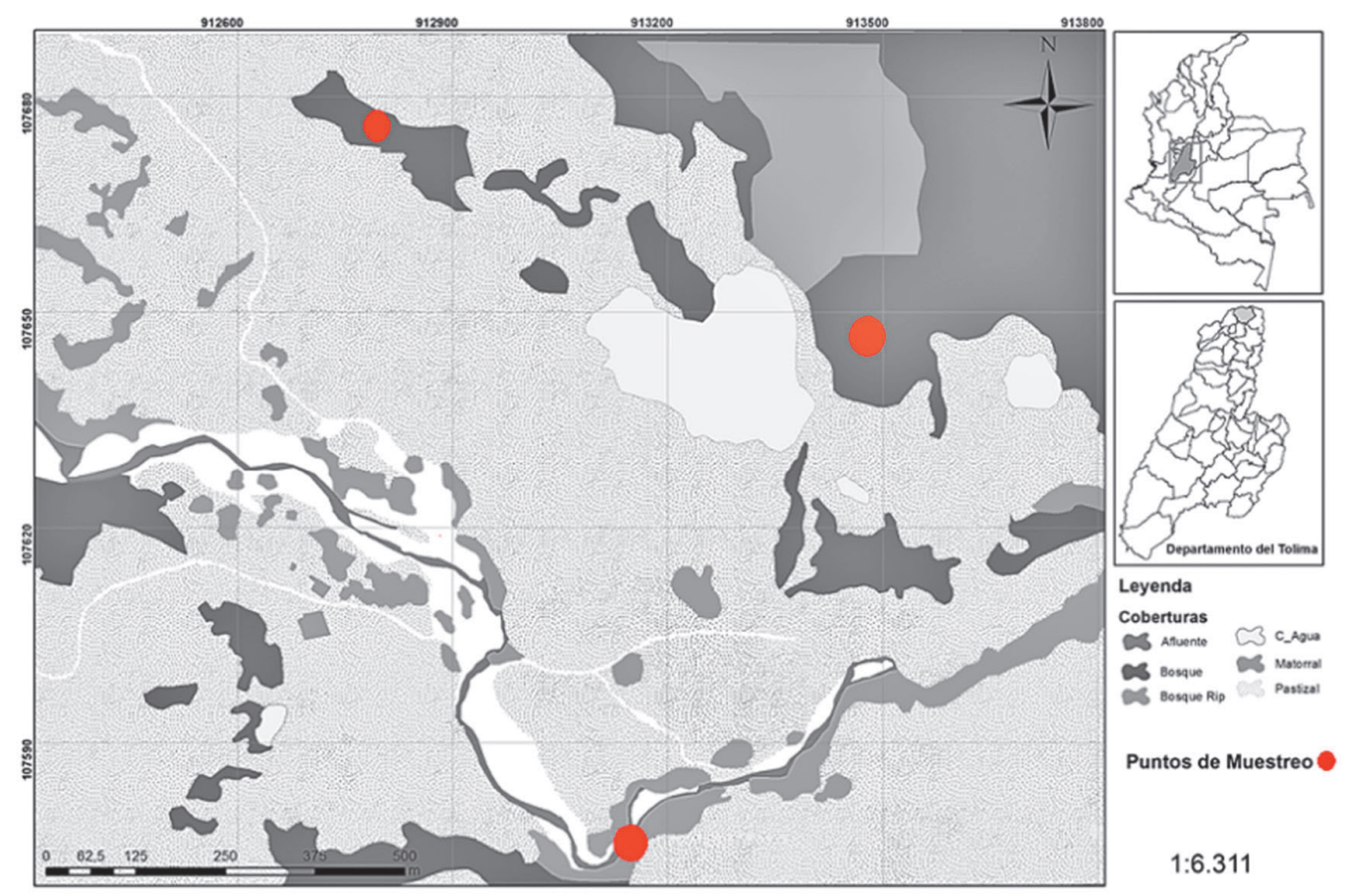

Figura 1. Ubicación del área de estudio. Los sitios de muestreo están representados por círculos de color rojo.

La captura de murciélagos filostómidos se realizó durante dos épocas contrastante (agosto y diciembre de 2010). Se instalaron seis redes de niebla de $12 \times 2,6 \mathrm{~m}$ en el sotobosque dentro del fragmento. Las redes se mantuvieron abiertas durante seis horas (18:00-24:00 h) y fueron revisadas a intervalos de 30 minutos. A cada murciélago capturado se le tomaron las medidas morfométricas estándar de acuerdo a Simmons y Voss [16] con un calibrador de precisión \pm 0.1 $\mathrm{mm}$ y pesado mediante una balanza electrónica de precisión $\pm 0.1 \mathrm{~g}$. La edad se determinó mediante el grado de osificación de las placas de crecimiento de la epífisis de las falanges de los dedos [17] y la condición reproductiva a partir de lo propuesto por Kunz [18].

Recolectamos al menos un espécimen de cada especie para confirmar la identificación a través de claves taxonómicas $[19,20]$ y construir una colección de referencia, todos los especímenes recolectados se depositaron en la Colección Zoológica de la Universidad del Tolima bajo los números de catálogo (CZUT 1054-1072).
Los individuos se trataron de acuerdo a las recomendaciones bioéticos propuestos por Sikes y Gannon [21].

Se colectaron muestras de heces al momento de la captura, además cada individuo fue depositado en bolsas de tela durante dos horas con el fin de colectar muestras fecales; estas se almacenaron en tubos Eppendorf para su separación y limpieza. La determinación taxonómica de las semillas se realizó en base a la colección de referencia del área de estudio, claves taxonómicas [22] y la asesoría del Laboratorio de Dendrología de la Universidad del Tolima

Para la descripción de la comunidad de murciélagos se calculó el esfuerzo de muestreo Ec (1)

\section{(1) $E m=m^{2} h$}

$\mathrm{m}^{2}=$ metro de red empleados.

$\mathrm{h}=$ horas efectivas de muestreo.

éxito de captura Ec (2) [23] 


\section{(2) $\mathrm{Ec}=\mathrm{Ni} / \mathrm{Em} * 100$}

$\mathrm{Em}=$ esfuerzo de muestreo

$\mathrm{Ni}=$ número de capturas

La eficiencia del muestreo se evaluó a través de la curva de acumulación de especies, empleado los datos observados y el modelo de Clench, mediante el software EstimateS 7.0 [24], modelo que permite encontrar el número esperado de especies, así como la proporción de la fauna muestreada.

La abundancia relativa (AR\%) se determinó a partir del número de individuos colectados de cada especie y su relación con el número total de individuos de la muestra. Este parámetro fue calculado con el fin de determinar la importancia y proporción en la cual se encuentra cada una de las especies respecto de la comunidad.

Con el fin de evaluar la composición trófica de la comunidad de murciélagos se consideró las categorías propuestas por Soriano [25], y para valorar la importancia de cada especie de murciélago frugívoro como agente de dispersor, se halló el índice de importancia del dispersor Ec (3) [7]
(3) $\mathrm{DII}=(\mathrm{S} * \mathrm{~B}) / 1000$

$\mathrm{S}=$ porcentaje de muestras fecales con semillas.

$\mathrm{B}=$ abundancia relativa de las especies de murciélagos capturados

Se determinó si la proporción de frutos consumidos por el conjunto de murciélagos diferían de forma significativa (0.05) mediante pruebas de chi-cuadrado $\left(\mathrm{x}^{2}\right)$, asimismo se describió la amplitud de la dieta mediante del índice de Levins (IL) [26], y se calculó la sobreposición de dietas a través del índice simplificado de Morisita $(\mathrm{CH})$ en el programa Ecological-Methodology, versión 7.01.

\section{Resultados y Discusión}

Con un esfuerzo total de muestreo de 2,620 $\mathrm{m}^{2}$ red*hora, y un éxito de captura de 3,3 individuos $/ \mathrm{m}^{2}$ red*h, se capturaron 85 individuos pertenecientes a tres subfamilias, siete géneros y 14 spp. De acuerdo con la curva de acumulación de especies y el modelo de Clench, se obtuvo una representatividad del ensamblaje de murciélagos mayor al 90\% (Figura 2).

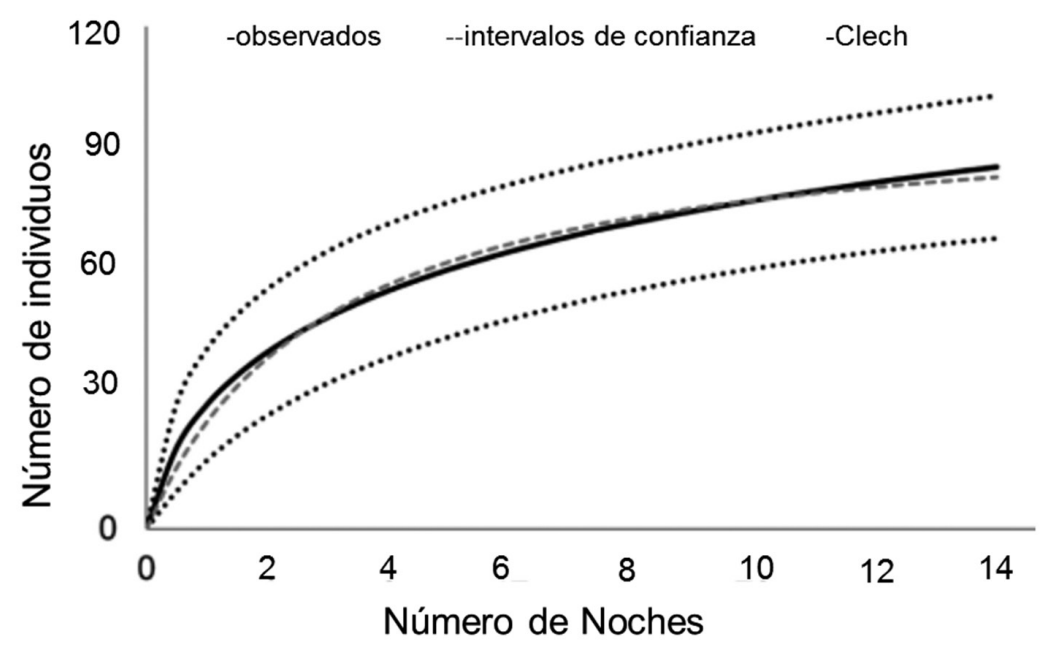

Figura 2. Curva de acumulación de especies. Línea negra continua muestra el número de especies observadas $(\mathrm{n}=14)$; líneas punteadas indican el intervalo de confianza al 95\%; línea gris discontinua índica el ajuste al modelo de Clench.

Las especies más frecuentes fueron: Carollia perspicillata con 16 individuos; lo que equivale al $18,82 \%$ de las capturas, seguida por Artibeus planirostris $(14 ; 16,4 \%)$ y Carollia brevicauda (11; 12,94\%). El hábito generalista de estas especies les permite adaptarse a las condiciones que el ambiente provea, explotando diferentes recursos [27]. Entre los murciélagos 
frugívoros neotropicales, C. perspicillata es una de las especies localmente más abundantes y ampliamente distribuida, se encuentra desde México hasta el norte de Argentina [28].

Los individuos capturados se agruparon en tres gremios tróficos, los frugívoros nómadas registraron la mayor proporción $(49,41 \%)$, seguido de los sedentarios $(41,18 \%)$ y los restantes se agruparon dentro de la categoría omnívora $(9,41 \%)$. La dominancia de frugívoros nómadas, podría atribuirse a una mayor facilidad de atravesar áreas abiertas, debido a que migran localmente en busca de plantas cuyos individuos se encuentran relativamente alejados unos de otros, con periodos de fructificación asincrónicos y asociados a hábitats con menor complejidad estructural. Este patrón es contrario a lo registrado en otros estudios, en donde se reportó una dominancia de murciélagos sedentarios [29, 30], situación que evidencia la fuerte transformación estructural del área de estudio.

A partir de las 86 muestras fecales analizadas, se encontraron semillas en 63 de ellas, lo que equivale al 73,25\%. El índice de dispersión (DII) permitió identificar que $C$. perspicillata $(0,21)$ y $A$. planirostris $(0,18)$ (Tabla 1$)$ contribuyeron con la mayor cantidad de muestras. Así mismo estas especies presentaron la mayor abundancia de semillas con $95(15,37 \%)$ y $119(19,09 \%)$. El índice de importancia de cada murciélago como dispersor mostró una dependencia de la abundancia sobre el papel que cumplen las especies de murciélagos como dispersores, encontrando que la mayoría de especies de frutos hacen parte de la dieta conocida de estos murciélagos, en donde predominan especies frutales pioneras del Neotrópico, como ha sido observado en otras regiones [31].

Tabla 1. índice de dispersión de semillas, Abundancia absoluta, abundancia relativa, y número de semillas evaluadas por especie de murciélago.

\begin{tabular}{lllcccc}
\hline \multicolumn{1}{c}{ Especie } & Gremio & DII & Abundancia & Ab-Rel & \# Muestras & \%-Mues \\
\hline Artibeus lituratus & Fru-Nom & 0.05 & 9 & 10.59 & 5 & 8.33 \\
Artibeus planirostris* & Fru-Nom & $\mathbf{0 . 1 8}$ & 14 & 16.47 & 9 & 15.00 \\
Carollia brevicauda & Fru-Sed & 0.14 & 11 & 12.94 & 11 & $\mathbf{1 8 . 3 3}$ \\
Carollia castanea & Fru-Sed & 0.06 & 5 & 5.88 & 2 & 3.33 \\
Carollia perspicillata* & Fru-Sed & $\mathbf{0 . 2 1}$ & 16 & $\mathbf{1 8 . 8 2}$ & 16 & $\mathbf{2 6 . 6 7}$ \\
Artibeus anderseni & Fru-Nom & 0.06 & 8 & 9.41 & 3 & 5.00 \\
Artibeus phaeotis & Fru-Nom & 0.02 & 3 & 3.53 & 2 & 3.33 \\
Mesophylla macconnelli & Fru-Nom & 0.00 & 2 & 2.35 & 1 & 1.67 \\
Phyllostomus discolor & Omn & 0.02 & 6 & 7.06 & 3 & 5.00 \\
Phyllostomus hastatus & Omn & 0.01 & 2 & 2.35 & 2 & 3.33 \\
Platyrrhinus helleri & Fru-Nom & 0.03 & 5 & 5.88 & 3 & 5.00 \\
Sturnira ludovici & Fru-Sed & 0.004 & 1 & 1.18 & 1 & 1.67 \\
Sturnira tildae & Fru-Sed & 0.004 & 2 & 2.35 & 1 & 1.67 \\
Uroderma bilobatum & Fru-Nom & 0.001 & 1 & 1.18 & 1 & 1.67 \\
\hline Total & & & $\mathbf{8 5}$ & & $\mathbf{6 0}$ &
\end{tabular}

*Especies con mayores abundancias dentro del presente estudio.

Fru-Nom: Frugívoro nómada. Fru-Sem: Frugívoro sedentario, Omn: Omnívoro DII: Índice de dispersión. Abun: Abundancia general. Ab-Rel: Abundancia relativa (\%).

\# Muestras: Número de muestras evaluadas. \%-Mues: Porcentaje por muestras evaluadas. 
Se hallaron un total de 11 especies de plantas dentro de la dieta de los murciélagos, tres géneros y una morfoespecie, agrupadas en ocho familias, donde Piperaceae y Solanaceae fueron las mejor representadas $(26,68 \%$ cada una), seguida de Asteraceae (16.8\%). A nivel de especies Bidens pilosa presentó el mayor número de registros $(15,9 \%)$, seguido de Piper crassinervium $(13,87 \%)$ y Physalis peruviana L. $(9,84 \%)$ (Tabla 2).

El aporte de cada especie de planta frente al porcentaje total de las muestras vario entre las dietas de los murciélagos. La dieta de A. lituratus estuvo constituida por una mayor cantidad de frutos (6 spp.), seguido por A. planirostris (4 spp.), mientras que Carollia castanea, Mesophylla macconnelli, Phyllostomus discolor, Sturnira ludovici, Sturnira tildae y Uroderma bilobatum registraron una sola especie de fruto en la dieta (Tabla 2).

La proporción de consumo de frutos de cada ítem alimentario fue significativamente diferente entre las 14 especies de murciélagos. $\left(X^{2}=74.15\right.$, $\mathrm{gl}=46, \mathrm{p}<0.05$ ), lo que evidencia una repartición de recursos; cada especie mostro una tendencia hacia ciertos géneros o especies de plantas, así Carollia presentó un mayor consumo de Bidens y Piper, mientras que el género Artibeus (grandes) por Solanum y Sturnira por Cecropia. Asociaciones que no siguen estrictamente las relaciones propuesta por sensu Fleming [32], Carollia-Piper, Sturnira-Solanum y Artibeus - Ficus-Cecropia. Estos registros añaden nuevos elementos a la dieta de estas especies. Es importante precisar que los murciélagos responden positivamente a cambios en la distribución y abundancia de frutos, de tal forma que puedan aprovechar la abundancia de los recursos, sin presentar una selectividad [25].

La mayor amplitud de dieta la registro $A$. lituratus $(\mathrm{IL}=8,52)$, seguido de $A$. planirostris $(\mathrm{IL}=8.2)$. Mediante el análisis de solapamiento de nicho alimentario, la mayor sobreposición de dieta la reportaron A. planirostris - A. lituratus $\left(\mathrm{C}_{\mathrm{H}}=0.42\right)$ y $C$. brevicauda $-C$. perspicillata $\left(\mathrm{C}_{\mathrm{H}}=0.36\right)$, no obstante, estos valores son inferiores al $50 \%$, lo que podría reflejar una segregación de nicho como mecanismo para evitar la competencia. Este patrón ha sido registrado en otras localidades del Neotrópico [33, 34], y constituye una base para generar hipótesis sobre las estrategias de separación de nicho entre especies simpátricas.

Los frutos de estado sucesional arbusto $(53,85 \%)$ estuvo representado ampliamente en la dieta de los murciélagos evaluados, seguido del estado subarbusto $(30,77 \%)$ y por último el estado árbol (15,38\%). Ello implica que cada estado sucesional tiene un ensamble de murciélagos que aprovechan sus recursos. Así, los géneros Artibeus (grandes) y Platyrrhinus preferencialmente consumieron frutos de subarbusto, mientras que Phyllotomus y Sturnira explotaron los recursos del estado árbol y por su parte los géneros Carollia y Artibeus (pequeños) presentaron un mayor consumo de frutos del estado arbusto (Tabla 2).

Tabla 2. Especies de plantas, forma de vida de las semillas encontrados en las muestras para cada especie de murciélago.

\begin{tabular}{lllcl}
\hline Familia de planta & Especie de planta & $\begin{array}{c}\text { Forma de } \\
\text { vida }\end{array}$ & $\begin{array}{c}\text { Abundancia } \\
\text { relativa }\end{array}$ & Especies de Murciélagos \\
\hline Asteraceae & Bidens pilosa & Arbusto & $\mathbf{1 5 . 9}$ & P. has, C. bre, C. per, A. plan, A. lit \\
Cecropiaceae & Cecropia telenitida & Árbol & 1.58 & S. lud, S. til \\
& Cecropia tealba & Árbol & 6.14 & A. lit, P. has, S. lud \\
Clusiaceae & Vismia guianensis & Arbusto & 4.04 & P. hell, S. lud \\
Euphorbiaceae & Phyllanthus niruri & Arbusto & 0.18 & S.lud, A. plan, A. lit \\
Gesneriaceae & Kohleria sp. & Arbusto & 7.72 & P. has, A. and \\
Piperaceae & Piper pesaresanum & Arbusto & 6.14 & C. bre, C. per,
\end{tabular}




\begin{tabular}{lllll} 
& Piper aduncum & Arbusto & 6.67 & C. bre, C. per, A. lit, S. til \\
Solanaceae & Solanum sp. & Subarbusto & 3.16 & A. plan, A. lit \\
& $\begin{array}{l}\text { Solanum quitoense. } \\
\text { Solanum cf. } \\
\text { quinquangulare }\end{array}$ & Subarbusto & 9.30 & A. plan, A. li \\
& $\begin{array}{l}\text { Physalis peruviana } \\
\text { L. }\end{array}$ & Subarbusto & $\mathbf{9 . 8 4}$ & A. pha, A. and,P. hell \\
& Urtica sp. & & & A. plan, A. lit \\
Urticaceae & M1. & & 8.43 & A. pha, A. and, P. hell \\
Morfotipo & & 1.76 & P. dis, A. lit, U. bil \\
\hline
\end{tabular}

A. lit: Artibeus lituratus; A. plan: Artibeus planirostris; C. bre: Carollia brevicauda; C. cas: Carollia castanea; C. per: Carollia perspicillata; A. and: Artibeus anderseni; A. pha: Artibeus phaeotis; P. dis: Phyllostomus discolor; P. has: Phyllostomus hastatus; P. hll: Platyrrhinus helleri; S. lud: Sturnira ludovici; S. til: Sturnira tildae; U. bil: Uroderma bilobatum

\section{CONCLUSIONES}

Nuestros resultados permiten confirman que los murciélagos filóstomidos centran su dieta en un grupo específico de plantas. Sin embargo, las interacciones no guardaron una relación estrecha con lo postulado por otros estudios. Por lo que sugerimos analizar con más detalle las variaciones de la dieta de estas especies y acompañarla con registros fenológicos para determinar los factores que pueden influir en la selección y consumo de frutos.

El ensamble de murciélagos evaluado incluyó en su dieta un alto número de plantas con diferentes formas de vida, lo que resalta la importancia de esta fauna para la persistencia de los fragmentos de bs-T en el departamento del Tolima.

\section{AGRADECIMIENTOS}

Agradecemos a los miembros del Laboratorio de Dendrología, especialmente a Boris Villanueva por la identificación de las semillas. A las Biólogas Emma Yicel Galindo y Karina Gutiérrez por su apoyo incondicional en la fase de campo y laboratorio, y a la Oficina de Investigaciones y Desarrollo Científico de la Universidad del Tolima, por la financiación de esta investigación y las dos primeras autoras agradecen a la Beca para adelantar los estudios doctorales otorgada por la Convocatoria para la Formación de Capital Humano de Alto Nivel para el Departamento de Tolima, No. 755, 2016 de Colciencias, tiempo en el cual se escribió este manuscrito.

\section{REFERENCIAS}

[1] A. Cubiña and T. M. Aide, "The effect of distance from forest edge on seed rain and soil seed bank in a tropical pasture", Biotropica, vol. 33, pp. 260-267, 2001.

[2] M. A. Aizen, D. Vázquez y C. Smith, "Historia natural y conservación de los mutualismos planta-animal del bosque templado de Sudamérica austral", Revista chilena de historia natural, vol. 75, pp. 7997, 2002.

[3] M. Romo, "Seasonal variation in fruit consumption and seed dispersal by canopy bats (Artibeus spp.) in a lowland forest in Peru", Vida Silvestre Neotropical, vol. 5, pp.110-119, 1996.

[4] T. Lobova, C. Geiselman and S. Mori, Seed dispersal by bats in the Neotropics. New York Botanical Garden Press, USA: New York, 2009.

[5] I. Vleut, J. Galindo-González, W. F. de Boer, S. L. Levy-Tacher and L. B. Vázquez, "Niche differentiation and its relationship with food abundance and vegetation complexity in four frugivorous bat species in Southern Mexico", Biotropica, vol. 47, no. 5, pp. 606-615, 2015. DOI.org/10.1111/ btp. 12238 
[6] M. C. de Carvalho-Ricardoa, W. Uiedab, R. C. Fonseca and M. N. Rossi, "Frugivory and the effects of ingestion by bats on the seed germination of three pioneering plants", Acta Oecologica, vol. 55, pp. 5551-5557, 2014.

[7] J. Galindo-González, S. Guevara and V. Sosa, "Bat and bird-generated seed rains at isolated trees in pastures in a tropical rainforest", Conservation Biology, vol. 14, no. 6, pp. 1693-1702, 2000. DOI. org/10.1111/j.1523-1739.2000.99072.x

[8] R. Dirzo, H.S. Young, H. Mooney and G. Ceballos, Seasonally Dry Tropical Forest; Ecology and Conservation. Island Press, USA: Washington, D.C., 2011.

[9] R. Dirzo, and P. H. Raven, "Global state of biodiversity and loss", Annual Review of Environmental Resources, vol. 28, pp. 137167, 2003

[10] C. A Portillo-Quintero and G. A. SánchezAzofeifa, "Extent and conservation of tropical dry forests in the Americas", Biological Conservation, vol. 143, pp. 144155, 2010.

[11] G. A. Sánchez-Azofeifa, M. Quesada, J. P. Rodríguez, J. M. Nassar, K. E. Stoner, A. Castillo, T. Garvin, E. L. Zent, J. C. CalvoAlvarado, M. E. R. Kalacska, L. Fajardo, J. A. Gamon, and P. Cuevas-Reyes, "Research priorities for Neotropical dry forests", Biotropica, vol. 37, pp. 477-485, 2005.

[12] M. Ríos-Blanco y J. Pérez-Torres, "Dieta de las especies dominantes del ensamblaje de murciélagos frugívoros en un bosque seco tropical (Colombia)", Mastozoología Neotropical, vol. 22, no 1, pp. 103-111, 2015.

[13] A. Aroca, L. González, M. Hurtado y O. Murillo-García, "Preferencia en la dieta de murciélagos frugívoros (Phyllostomidae) en un fragmento de bosque seco tropical", Revista de Ciencias, vol. 20, pp. 139-146, 2016.
[14] A. Suarez-Castro y O. Montenegro, "Consumo de plantas pioneras por murciélagos frugívoros en una localidad de la Orinoquía colombiana", Mastozoología Neotropical, vol. 22, no 1, pp. 125-139, 2015.

[15] Corporación Autónoma y Regional del Tolima (CORTOLIMA), Caracterización ambiental del municipio de Mariquita. Plan de ordenamiento territorial, Ibagué, Colombia, 1997.

[16] N. B. Simmons, and R. S. Voss, "The mammals of Paracou, French Guiana: A Neotropical lowland rainforest fauna", Bulletin of the American Museum of Natural History, vol. 237, pp. 1-219, 1998.

[17] C. Dietz, O. Von Helversen, and D. Nill, Bats of Britain, Europe \& Northerwest Africa. A \& C Black. London. 2009.

[18] T.H. Kunz, Methods of marking bats, in Measuring and monitoring biological diversity standard methods for mammals. Smithsonian Institution (Wilson, D. E. J. Nichols, R. Rudrin, R. Cole y M. Foster, eds.). Washington D.C., USA, pp. 304-310, 1996.

[19] A. Gardner, "Mammals of South America Volume 1. Marsupials, Xenarthrans, Shrews, and Bats". The University of Chicago Press. Chicago, 2008.

[20] M. M. Díaz, S. Solari, L. F. Aguirre, I. Aguiar y R. M. Barquez, "Clave de identificación de los murciélagos de Sudamérica /Chave de indentifição dos morcegos da América do Sul". Publicación Especial PCMA Nro 2, Editorial Magna Publicaciones, Argentina, 2016.

[21] R. S. Sikes, W. H. Gannon, and the animal care and use committee of the American Society of Mammalogists, "Guidelines of the American Society of Mammalogists for the use of wild mammals in research and education", Journal of Mammalogy, vol. 97, pp. 663-688, 2011. 
[22] F. Cornejo, and J. Janovec, Seeds of Amazonian plants. Princeton University Press, USA: Princeton, 2010.

[23] F.C. Straube, and G.V. Bianconi, "Sobre a grandeza e a unidade utilizada para estimar esforço de captura com utilização de redesde-neblina", Chiroptera Neotropical, vol. 8, pp. 150-152, 2002.

[24] R. K. Colwell, EstimateS: Statistical Estimation of Species Richness and Shared Species from Samples (Software and User's Guide), Versión 7.01, 1997. http://viceroy. eeb.uconn.edu/estimates.

[25] P. Soriano, "Functional structure of bat communities in tropical rainforests and andean cloud forests", Ecotrópicos, vol. 1, pp. 1-20, 2000.

[26] M. S. Sánchez, N. P. Giannini and R. M. Barquez, "Bat frugivory in two subtropical rainforest of Northern Argentina: Testing hypotheses of fruit selection in the in the Neotropics", Mammalian Biology, vol. 77, pp. 22-31, 2012.

[27] E. K. Kalko y C. O. Handley, "Neotropical bats in the Canopy: diversity, community structure, and implications for conservation”, Plant Ecology, vol. 153, pp. 319-333, 2001.

[28] O. Murillo-García, "Murciélagos de cola corta (Carollia: Phyllostomidae) del Parque Nacional Natural Gorgona (Colombia) y sus implicaciones biogeográficas", Revista de Biología Tropical, vol. 62, pp. 435-445, 2014.
[29] J. Galindo-Gonzáles, "Dispersión de semillas por murciélagos: su importancia en la conservación y regeneración del bosque tropical", Acta Zoológica Mexicana, vol. 73, pp. 57-74, 1998.

[30] M. Shulze, N. Seavy and D. Whitacre, "A comparison of the Phyllostomid bat assemblages in undisturbed Neotropical an in forest fragments of a slash-and-burn farming mosaic in Petén, Guatemala", Biotropica, vol. 32, pp. 174-184, 2000.

[31] A. Olea-Wagner, C. Lorenzo, E. Naranjo, D. Ortiz y L. León-Paniagua, "Diversidad de frutos que consumen tres especies de murciélagos (Chiroptera: Phyllostomidae) en la selva Lacandona, Chiapas, México", Revista Mexicana de Biodiversidad, vol. 78, pp. 191-200, 2007.

[32] T. H. Fleming, "Opportunism versus specialization: the evolution of feeding strategies in frugivorous bats", in Frugivores and seed dispersal, A. Estrada, T. H. Fleming eds., W. Junk Publishers, Netherlands, 1986, Pp. 105-118.

[33] R. A. Saldaña-Vázquez, V. J. Sosa, L. Iñiguez-Dávalos and J. E. Schondube. "The role of extrinsic and intrinsic factors in Neotropical fruit bat-plant interactions", Journal of Mammalogy, vol. 94, pp. 632639, 2013.

[34] T. Y. Andrade, W. Thies, P. K. Rogeri, E. K. Kalko and M. A. Mello, "Hierarchical fruit selection by Neotropical leaf-nosed bats (Chiroptera: Phyllostomidae)", Journal of Mammalogy, vol. 94, pp. 1094-1101, 2013. 